\title{
Torque Detection using Brownian Fluctuations
}

\author{
Giovanni Volpe ${ }^{1}$ and Dmitri Petrov ${ }^{1,2, *}$ \\ ${ }^{1}$ ICFO - Institut de Ciencies Fotoniques, Mediterranean Technology Park, 08860, Castelldefels (Barcelona), Spain \\ ${ }^{2}$ ICREA - Institució Catalana de Recerca i Estudis Avançats, 08010, Barcelona, Spain \\ (Received 27 July 2006; published 22 November 2006)
}

\begin{abstract}
We report the statistical analysis of the movement of a submicron particle confined in a harmonic potential in the presence of a torque. The absolute value of the torque can be found from the auto- and cross-correlation functions of the particle's coordinates. We experimentally prove this analysis by detecting the torque produced onto an optically trapped particle by an optical beam with orbital angular momentum.
\end{abstract}

The way in which deterministic perturbations affect the random walk of a small particle immersed in a fluid bath (Brownian motion) underlies many physical, chemical, and biological phenomena: examples include Brownian motors [1], molecular transport processes [2], thermally activated transitions in a potential landscape [3], and thermal fluctuations in an optical trap [4]. In a harmonic potential well, a Brownian particle oscillates around the equilibrium position. A statistical analysis of these fluctuations allows one to measure pico- to femto-Newton forces [5-11]. Another type of perturbation is a mechanical torque which can be exerted on the Brownian particle. This has previously been studied by measuring the rotation rate of the particle itself [12-22] and a statistical description is available for the case of rotational Brownian motion (the Brownian movement of the particle around its own axis) [23]. However, the precise manner in which the torque affects the statistics of the translational Brownian trajectories (the Brownian movement of the center of mass of the particle) is still unknown. Here we report the analysis and measurements of the movement of a submicron particle confined in a harmonic potential in the presence of a torque. The absolute value of the torque can be found from the auto- and cross-correlation functions of the particle's coordinates. We anticipate our study to be a starting point for the development of new techniques to measure the torque produced, for example, by biomolecules, molecular motors, or by optical beams with angular momentum.

We consider a sphere of mass $m$ and radius $R$ suspended in a liquid medium and confined within a harmonic potential well, where it moves randomly due to the thermal excitation [Fig. 1(a)]. We suppose an external torque is exerted on the sphere. In the absence of the potential well due to the friction the sphere rotates around the $z$ axis with a constant angular velocity $\Omega$, whose value results from a balance between the torque applied to the sphere and the drag torque: $\boldsymbol{\tau}_{\text {drag }}=\mathbf{r} \times \mathbf{F}_{\text {drag }}=\gamma \mathbf{r} \times \mathbf{v}=\gamma \mathbf{r} \times(\mathbf{r} \times$ $\boldsymbol{\Omega})$, where $\mathbf{r}$ is the sphere's position, $\mathbf{v}$ is its linear velocity, $\gamma=6 \pi R \eta$ is the friction coefficient, and $\eta$ is the viscosity. Hence, the force acting on the sphere from the torque source is given by $\mathbf{F}=\gamma \mathbf{r} \times \Omega$, which depends on the position of the sphere. A time average of the torque exerted on the particle can then be expressed as $\langle\tau\rangle=\gamma\langle\mathbf{r} \times(\mathbf{r} \times$ $\Omega)\rangle=\gamma\left\langle\Omega r^{2}\right\rangle=\gamma \Omega\left\langle r^{2}\right\rangle$, where $\left\langle r^{2}\right\rangle$ is the mean square displacement of the sphere in the plane orthogonal to the torque.

The Einstein-Ornstein-Uhlenbeck equations [24] for the Brownian motion of the sphere in the plane perpendicular to the rotation axis can now be presented as:

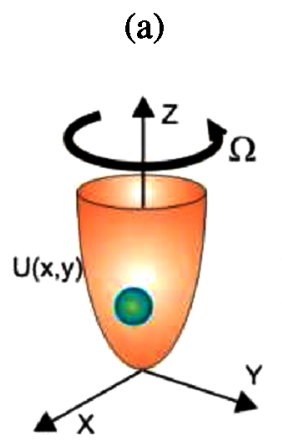

(c)

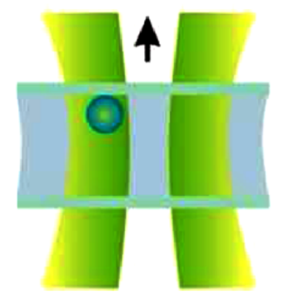

(b)

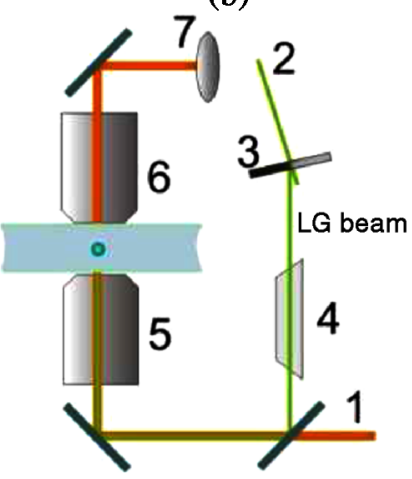

(d)

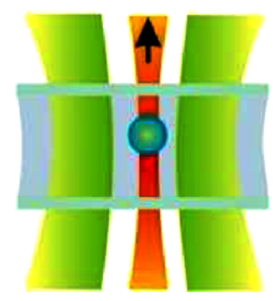

FIG. 1 (color online). (a) Harmonic potential well $U(x, y)=$ $\frac{k}{2}\left(x^{2}+y^{2}\right)$ ( $k$ is the restoring force constant or stiffness of the harmonic oscillator) with a Brownian particle inside and with an external torque acting on the particle. (b) Experimental setup: 1 -trapping $785 \mathrm{~nm}$ laser beam, 2-532 nm beam, 3-holographic mask, $4-$ Dove prism, 5-100 $\times 1.3 N A$ objective, $6-$ collimating $40 \times$ objective, 7-quadrant photodetector. (c) Position of the sphere in the chamber when only the $532 \mathrm{~nm}$ LG propagates, and (d), when both the $532 \mathrm{~nm}$ and the $785 \mathrm{~nm}$ beams propagate in the chamber. The arrows show the propagation direction of the trapping and LG beams. 


$$
\begin{aligned}
& \gamma \frac{d x(t)}{d t}+k x(t)+\gamma \Omega y(t)=\sqrt{2 k_{B} T \gamma} \eta_{x}(t), \\
& \gamma \frac{d y(t)}{d t}+k y(t)-\gamma \Omega x(t)=\sqrt{2 k_{B} T \gamma} \eta_{y}(t),
\end{aligned}
$$

where $k$ is the force constant of the harmonic oscillator and $\sqrt{2 k_{B} T} \bar{\gamma} \eta_{x}(t)$ and $\sqrt{2 k_{B} T \gamma} \eta_{y}(t)$ are two independent white Gaussian random processes that represent the Brownian forces at temperature $T$ in the $x$ and $y$ direction. To simplify the problem we set the rotation axis coaxial to the axis of the potential well. Assuming a low Reynolds number regime $[25,26]$, we can neglect all inertial terms such as $m \frac{d^{2} y(t)}{d t^{2}}$.

The terms $+\gamma \Omega y(t)$ and $-\gamma \Omega x(t)$ introduce a coupling between the equations. The auto- and cross-correlation functions for the movement of the sphere along the $x$ and $y$ directions are given by:

$$
\begin{aligned}
\langle x(t) x(t+\Delta t)\rangle & =\langle y(t) y(t+\Delta t)\rangle \\
& =\frac{k_{B} T}{k} e^{-k|\Delta t| / \gamma} \cos (\Omega \Delta t), \\
\langle x(t) y(t+\Delta t)\rangle & =\frac{k_{B} T}{k} e^{-k|\Delta t| / \gamma} \sin (\Omega \Delta t) .
\end{aligned}
$$

Accordingly, a change is produced also in the power spectral density (PSD) of the $x$ and $y$ coordinates, which, as well as the autocorrelation function, is normally employed in the calibration of the photonic force microscope $[4,26,27]$. Hence, the presence of a torque has to be taken into account in the calibration of force constant of the trap and in the force measurement.

The actual shape of the correlation functions depends on the ratio $\gamma \Omega / k$. For $\gamma \Omega<k$ the restoring force induced by the potential well is dominant and at the limit $\gamma \Omega \ll k$ the autocorrelation function is reduced to the known expression for the behavior of a Brownian particle in a harmonic potential [27], while the cross correlation is negligible. The correlation decay time is $t_{0}=\gamma / k$ and its measurement allows one to determine the force constant of the potential well. For $\gamma \Omega>k$ the rotational effect is dominant; at the limit $\gamma \Omega \gg k$ we get the sinusoidal auto- and crosscorrelation functions describing a rotating particle.

Both these limiting cases have already been studied: namely, when no external torque is applied to the Brownian particle in the parabolic potential (see, for example, Refs. [4-10] ) or when the torque is applied to a free particle and can be straightforwardly measured from the particle rotation rate assessed by video or Fourier analysis [12,14-18,28]. Equations (3) and (4) bridge these two cases and provide new insights into the intermediate situation, which neither of the cases can address. As we show below the analysis of the auto- and cross-correlation functions allows us to gain sensitivity over the methods based on the explicit measurement of the rotation rate.
For an experimental verification of our conclusions, we have analyzed the Brownian motion of an optically trapped polystyrene sphere. A focused beam creates a harmonic trapping potential as a result of electric field gradient forces exerted on the sphere. This optical trap is generated by a cw $785 \mathrm{~nm}$ beam at the focal plane of a $100 \times 1.3 \mathrm{NA}$ objective lens inside a chamber [Fig. 1(b)]. The chamber is prepared using two cover slips separated by a $50 \mu \mathrm{m}$ spacer and filled with a solution containing polystyrene spheres (radius $R=0.5 \mu \mathrm{m}$ ). The forward scattered light from the trapped sphere is collimated by a $40 \times$ objective onto a quadrant photodiode (QPD). The trap force constant $k$ can be adjusted by changing the intensity of the laser beam.

We induce a torque on the particle by using another optical beam that carries an orbital angular momentum [20,29-31]. This beam is produced from a linearly polarized $532 \mathrm{~nm} \mathrm{cw}$ beam transformed by a holographic mask that generates a $l=10$ order Laguerre-Gaussian (LG) beam of power $P=3 \mathrm{~mW}$. The beam carries the flux of orbital angular momentum $\tau_{\mathrm{OAM}}=l P / \omega=7.5 \times$ $10^{-18} \mathrm{Nm}$, where $\omega=4 \times 10^{15} \mathrm{~Hz}$ is the light frequency. Only about $1 \%$ of the total flux of the orbital angular momentum is transferred to the dielectric sphere [21] and only in the portion of the beam that overlaps the sphere. We estimate that in our experiments the beam transfers the torque $\tau_{S}=7.5 \times 10^{-21} \mathrm{Nm}$ to the sphere. A Dove prism can be inserted in the optical path so as to reverse the handedness of the helical phase front and therefore the sign

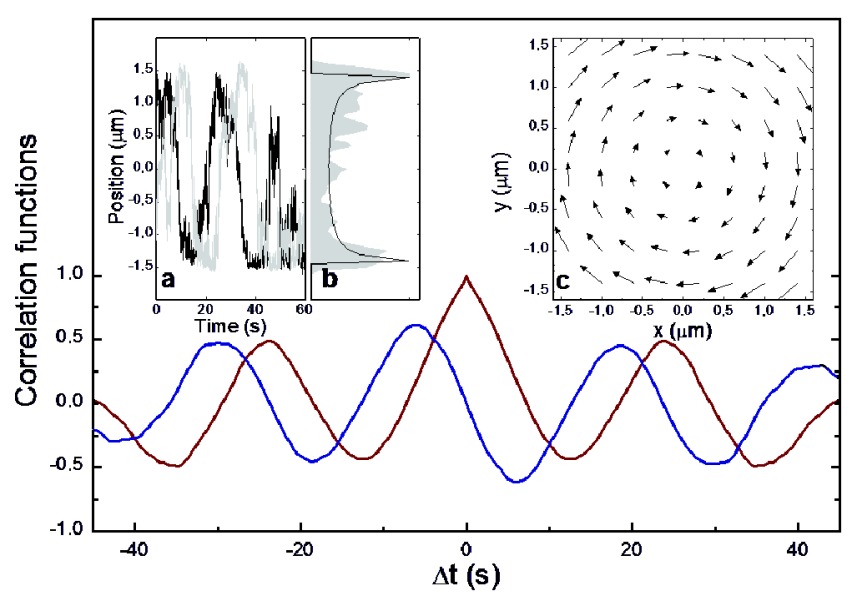

FIG. 2 (color online). Experimental unbiased auto- and crosscorrelation functions in the presence of the torque induced by a LG beam with $l=+10$. The trap force constant $k$ is low enough $(0.9 \mathrm{fN} / \mu \mathrm{m})$ not to significantly influence the rotational motion of the sphere. The continuous lines show the mean values obtained using one series of data acquisition (acquisition time $60 \mathrm{~s}$, sampling rate $f_{s}=1 \mathrm{kHz}$ ). In the insets: (a) time traces for the $x$ (black) and $y$ (gray) coordinates; (b) histogram of the $x$ coordinate; (c) vector force field acting on the particle in the $x y$ plane. 
of the orbital angular momentum without changing the direction or polarization of the beam.

In the absence of the $785 \mathrm{~nm}$ optical trap [Fig. 1(c)] the $532 \mathrm{~nm}$ beam moves the sphere towards the upper cover slip due to its radiation pressure and the gradient forces in the perpendicular plane do not allow the sphere to escape. In such conditions we can observe the clockwise and anticlockwise rotation of the sphere depending on the handedness of the beam.

When the trapping $785 \mathrm{~nm}$ beam coaxial to the LG beam is switched on and the trap force constant $k$ is low enough $(0.9 \mathrm{fN} / \mu \mathrm{m})$, the rotation motion of the sphere is not significantly influenced. We can track the position of the particle with the QPD and calculate the corresponding auto- and cross-correlation functions (Fig. 2). The rotation is clearly visible in the time traces [Fig. 2(a)] and from the position probability density function [Fig. 2(b)] which is not Gaussian in this case and it can be readily observed in the Fourier spectra of the traces, as was reported earlier [16]. The presence of the periodical component in the correlation functions is consistent with Eqs. (3) and (4) and permits us to measure the mean rotation rate of the bead and the torque exerted on it. We found the rotation period to be $T_{r}=20 \pm 5 \mathrm{~s}$ and an orbital radius of $r=$ $1.4 \pm 0.3 \mu \mathrm{m}$, in agreement with the video data. This corresponds to a torque produced by the beam of $\tau_{r}=$ $\gamma \Omega r^{2}=3.9 \pm 1.8 \times 10^{-21} \mathrm{Nm}$ when the friction coefficient, corrected for the distance to the surface [26], is $\gamma=$ $4 \pm 1 \times 10^{-8} \mathrm{~N} \mathrm{~s} / \mathrm{m}$. The experimental numerical values are expressed as mean \pm standard error and are obtained from three series of data. Figure 2(c) shows the measured vector force-field acting on the particle, where the azimuthal component due to the applied torque is dominant.

We now change the conditions of the experiment, namely, by increasing the trap stiffness, to verify whether the effect of the torque on the Brownian motion of the sphere can still be retrieved. The trapping $785 \mathrm{~nm}$ beam power and therefore the trap stiffness are increased $(100 \mu \mathrm{W}, 16 \mathrm{fN} / \mu \mathrm{m})$; the sphere is more confined in the center of the trapping beam [Fig. 1(d)] and does not display a rotational motion, as can be readily appreciated from the time traces [Fig. 3(a)] and from the position probability density function [Fig. 3(b)] which is now Gaussian. We notice that in this case the Fourier analysis of the experimental traces does not show the presence of the torque existing in the system. However, the behavior of the auto- and cross-correlation functions near $\Delta t=0$ (Fig. 3) unambiguously shows that the torque produced by the orbital angular momentum of the LG beam still affects the Brownian trajectories. Fitting these experimental functions to the theoretical ones and calculating the value of $\left\langle r^{2}\right\rangle$ from the traces, we are able to measure the torque acting on the particle as $4.9 \pm 0.7 \times 10^{-21} \mathrm{Nm}$. Figure 3(c) depicts the vector force field acting on the particle, which results from the superposition of a azimu-

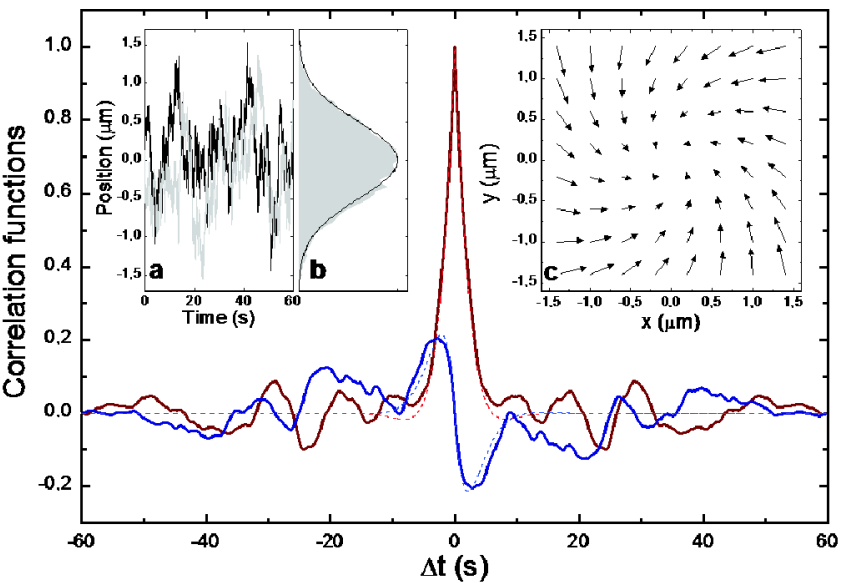

FIG. 3 (color online). Experimental auto- and crosscorrelation functions in presence of the torque induced by a LG beam with $l=+10$. The trap force constant $k$ is high enough (16 fN/ $/ \mu \mathrm{m})$ to confine the sphere. The continuous lines show the mean values obtained using five series of data acquisition (acquisition time $60 \mathrm{~s}$, sampling rate $f_{s}=1 \mathrm{kHz}$ ). The dotted lines show the fitting to the theoretical shape (the fitting was made on the central part of the curve for $\Delta t=[-2 \mathrm{~s}, 2 \mathrm{~s}]$ ). In the insets: (a) time traces for the $x$ (black) and $y$ (gray) coordinates; (b) histogram of the $x$ coordinate and in black the fitting to a Gaussian distribution; (c) vector force field acting on the particle in the $x y$ plane.

thal component due to the applied torque and a radial component due to the harmonic trap. The same procedure was repeated for the other handedness of the LaguerreGaussian beam (Fig. 4) leading to a torque of $3.9 \pm 0.8 \times$ $10^{-21} \mathrm{Nm}$. The lower value can be explained by the power losses in the Dove prism. The presence of the torque also affects the calibration of the trap force constant. Indeed, the value of $k$ found from the experimental traces accounting for the torque has the value $16 \pm 1 \mathrm{fN} / \mu \mathrm{m}$, while not accounting for the torque it is $18 \pm 1 \mathrm{fN} / \mu \mathrm{m}$. The experimental values are expressed as mean \pm standard error and are obtained from five independent series of data.

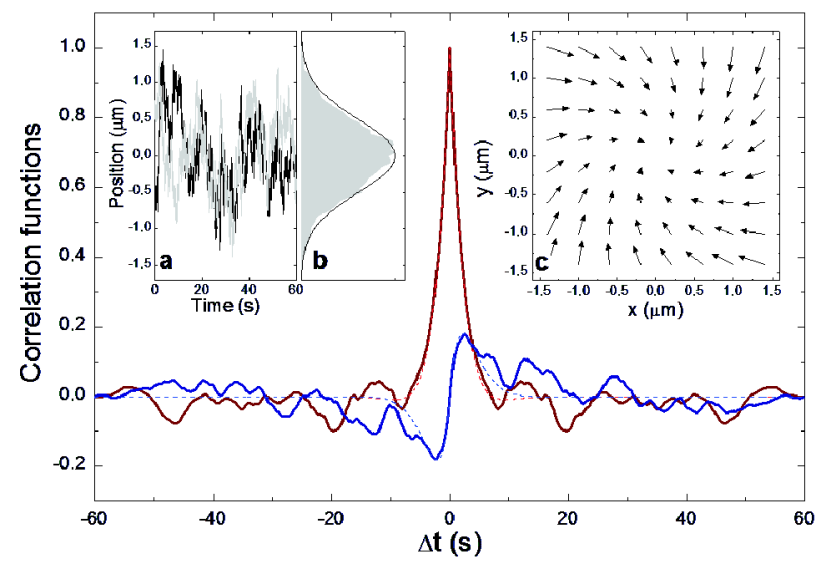

FIG. 4 (color online). The same as in Fig. 2 when the torque is produced by a LG with $l=-10$. 
The value of the torques measured in our experiments $\left(4 \times 10^{-21} \mathrm{Nm}\right)$ is lower than the ones previously reported, e.g., $10^{-20} \mathrm{Nm}$ for DNA twist elasticity [15], $5 \times$ $10^{-18} \mathrm{Nm}$ for the movement of bacterial flagellar motors [12], $2 \times 10^{-20} \mathrm{Nm}$ for the transfer of orbital optical angular momentum [21], or $5 \times 10^{-19} \mathrm{Nm}$ for the transfer of spin optical angular momentum [18]. Hence, as we have shown, a detailed analysis of the Brownian fluctuations of a particle trapped in a harmonic potential may be a starting point to build new tools for the measurement of torque in micrometric systems, like those produced by biomolecules, hydrodynamic interactions between colloidal particles, or by optical beams that carry orbital angular momentum. The restoring force acting on the Brownian particle depends on the trapping power. Hence, this could make it possible to study how the torque exerted by a certain source varies in the presence of a controlled mechanical load. We also notice that the presence of a torque significantly affects the correlation functions of the Brownian particle in the potential well so that experimental results previously obtained to measure only mechanical forces could reveal the presence of torques in the studied systems if analyzed using Eqs. (3) and (4).

We thank Giorgio Volpe for numerical simulations, Lluís Torner and Maciej Lewenstein for helpful discussions, and Gabriel Molina-Terriza for providing us the holographic masks. This research was carried out in the framework of ESF/PESC (Eurocores on Sons), through Grant No. 02-PE-SONS-063-NOMSAN, and with the financial support of the Spanish Ministry of Education and Science. It was also partially supported by the Departament d'Universitats, Recerca i Societat de la Informació and the European Social Fund.

*Electronic address: Dmitri.Petrov@icfo.es

[1] R. D. Astumian, Science 276, 917 (1997).

[2] R. F. Fox, Phys. Rev. E 57, 2177 (1998).

[3] L. I. McCann, M. Dykman, and B. Golding, Nature (London) 402, 785 (1999).

[4] L. Ghislain and W. W. Webb, Opt. Lett. 18, 1678 (1993).

[5] K. Svoboda, C. F. Schmidt, B. J. Schnapp, and S. M. Block, Nature (London) 365, 721 (1993).

[6] A. D. Menta, M. Rief, J. A. Spudich, D. A. Smith, and R. M. Simmons, Science 283, 1689 (1999).

[7] D. E. Smith, S. J. Taus, S. B. Smith, S. Grimes, D. L. Anderson, and C. Bustamante, Nature (London) 413, 748 (2001).
[8] M. J. Lang, C. L. Asbury, J. W. Shaevitz, and S. M. Block, Biophys. J. 83, 491 (2002).

[9] S. B. Smith, Y. Cui, and C. Bustamante, Method. Enzymol. 361, 134 (2003).

[10] A. Rohrbach, Opt. Express 13, 9695 (2005).

[11] G. Volpe, R. Quidant, G. Badenes, and D. Petrov, Phys. Rev. Lett. 96, 238101 (2006).

[12] R. M. Berry and H. C. Berg, Proc. Natl. Acad. Sci. U.S.A. 94, 14433 (1997).

[13] N. B. Simpson, K. Dholakia, L. Allen, and M. J. Padjett, Opt. Lett. 22, 52 (1997).

[14] W. S. Ryu, R. M. Berry, and H. C. Berg, Nature (London) 403, 444 (2000).

[15] Z. Bryant, M.D. Stone, J. Gore, S. B. Smith, N. R. Cozzarelli, and C. Bustamante, Nature (London) 424, 338 (2003).

[16] A. D. Rowe, M. C. Leake, H. Morgan, and R. M. Berry, J. Mod. Opt. 50, 1539 (2003).

[17] A. I. Bishop, T. A. Nieminen, N. R. Heckenberg, and H. Rubinsztein-Dunlop, Phys. Rev. A 68, 033802 (2003).

[18] A. La Porta and W. D. Wang, Phys. Rev. Lett. 92, 190801 (2004).

[19] M. Capitanio, D. Normanno, and F. S. Pavone, Opt. Lett. 29, 2231 (2004).

[20] V. Garcés-Chávez, D. McGloin, M. J. Padgett, W. Dultz, H. Schmitzer, and K. Dholakia, Phys. Rev. Lett. 91, 093602 (2003).

[21] K. Volke-Sepulveda, V. Garcés-Chávez, S. Chávez-Cerda, J. Arlt, and K. Dholakia, J. Opt. B 4, S82 (2002).

[22] S. Parkin, G. Kröner, T. A. Nieminen, N. R. Heckenberg, and H. Rubinsztein-Dunlop, Opt. Express 14, 6963 (2006).

[23] W. T. Coffey, J. Phys. D: Appl. Phys. 10, L83 (1977).

[24] M.C. Wang and G. E. Uhlenbeck, Rev. Mod. Phys. 17, 323 (1945).

[25] E. M. Purcell, Am. J. Phys. 45, 3 (1977).

[26] K. Berg-Sørensen and H. Flyvbjerg, Rev. Sci. Instrum. 75, 594 (2004).

[27] R. Bar-Ziv, A. Meller, T. Tlutsy, E. Moses, J. Stavans, and S. A. Safran, Phys. Rev. Lett. 78, 154 (1997).

[28] J. Gore, Z. Bryant, M. D. Stone, M. Nollmann, N. R. Cozzarelli, and C. Bustamante, Nature (London) 439, 100 (2006).

[29] L. Allen, M. W. Beijersbergen, R. J. C. Spreeuw, and J. P. Woerdman, Phys. Rev. A 45, 8185 (1992).

[30] H. He, M.E.J. Friese, N.R. Heckenberg, and H. Rubinsztein-Dunlop, Phys. Rev. Lett. 75, 826 (1995).

[31] L. Paterson, M. P. MacDonald, J. Arlt, W. Sibbett, P.E. Bryant, and K. Dholakia, Science 292, 912 (2001). 